University of Wollongong

Research Online

Faculty of Engineering and Information

Faculty of Engineering and Information

Sciences - Papers: Part A

Sciences

$1-1-2015$

\title{
A new approach to reduce the expected energy not supplied in a power plant located in a non-expandable transmission system
}

Hadi Lomei

University of Wollongong, hl123@uowmail.edu.au

Danny Sutanto

University of Wollongong, soetanto@uow.edu.au

Kashem M. Muttaqi

University of Wollongong, kashem@uow.edu.au

Mohsen Assili

Shahrood University of Technology

Follow this and additional works at: https://ro.uow.edu.au/eispapers

Part of the Engineering Commons, and the Science and Technology Studies Commons

Research Online is the open access institutional repository for the University of Wollongong. For further information contact the UOW Library: research-pubs@uow.edu.au 


\title{
A new approach to reduce the expected energy not supplied in a power plant located in a non-expandable transmission system
}

\begin{abstract}
The main objective of most of power plants is to inject power as much as possible into the grid from the power plants. However, the transmission system may restrain the output capability of the power plant, especially, if the power plant is located in an area of non-expandable transmission system. In this situation, any disturbance in the nearby transmission system may force the plant to generate lower than its rated value, which disables it from selling the remaining available generated power to the costumers and also increases the cost of energy not supplied. In this paper, an efficient method is proposed to determine the maximum output capacity of the power plant, by which the avoidable cost of energy not supplied, is not burdened on the plant. The effectiveness of the proposed method has been evaluated on a three machine test system and also on the actual large Mashhad power plant in Iran.
\end{abstract}

\section{Keywords}

transmission, expandable, non, located, plant, power, supplied, not, energy, system, expected, approach, reduce

\section{Disciplines}

Engineering | Science and Technology Studies

\section{Publication Details}

H. Lomei, D. Sutanto, K. M. Muttaqi \& M. Assili, "A new approach to reduce the expected energy not supplied in a power plant located in a non-expandable transmission system," in Power Engineering Conference (AUPEC), 2015 Australasian Universities, 2015, pp. 1-6. 


\section{A New Approach to Reduce the Expected Energy Not Supplied in a Power Plant Located in a Non- Expandable Transmission System}

\author{
Hadi Lomei*, Danny Sutanto, Kashem M. Muttaqi \\ School of Electrical, Computer and Telecommunications \\ Engineering, University of Wollongong \\ Wollongong, Australia \\ Email: *hl123@uow.edu.au
}

\author{
Mohsen Assili \\ Department of Electrical and Robotic Engineering, \\ Shahrood University of Technology \\ Shahrood, Iran
}

\begin{abstract}
The main objective of most of power plants is to inject power as much as possible into the grid from the power plants. However, the transmission system may restrain the output capability of the power plant, especially, if the power plant is located in an area of non-expandable transmission system. In this situation, any disturbance in the nearby transmission system may force the plant to generate lower than its rated value, which disables it from selling the remaining available generated power to the costumers and also increases the cost of energy not supplied. In this paper, an efficient method is proposed to determine the maximum output capacity of the power plant, by which the avoidable cost of energy not supplied, is not burdened on the plant. The effectiveness of the proposed method has been evaluated on a three machine test system and also on the actual large Mashhad power plant in Iran.
\end{abstract}

Index Terms--contingency analysis; energy not supplied; load duration curve; sensitive lines.

\section{INTRODUCTION}

Most of the restrictions on electric power transfers are directly related to the reliability of power system. Investigation of power system reliability clearly involves a consideration of system states and whether they are adequate or secure. This is particularly true in the case of transmission systems. The concept of adequacy is generally considered to be the existence of sufficient power plants within the system to satisfy the load demand, while security is associated with the response of the system to whatever disturbances they are subjected to. These include conditions causing local and widespread effects due to the loss of major generation and transmission facilities [1].

Over the past two decades, different quantitative transmission system reliability techniques have been developed to accurately reflect the stochastic nature of a power-system behavior to assess its reliability performance [2]. Moreover in [3], Monte Carlo method is also used to assess the reliability of power system. An approach to determine an acceptable limit for power system reliability and considering it as a constraint for future power system planning strategies is presented in [4]-[8]. In addition, the amount of spinning reserve requirement of the generating unit is also determined to minimize the cost of generation and the load demand which is not supplied [9]-[11].
Different methods [12]-[21] have been reported to perform contingency analysis for power system reliability. In almost all of these methods, power system equipment outage analysis is used to perform the contingency analysis [12]-[18]. In some cases, voltage stability is evaluated using contingency analysis of transmission lines and then each contingency is ranked according to its influence on the voltage level of the bus-bars [14], [18]-[21]. In [15], the influence of the line outage contingency on the loading level of network transmission lines has been studied. An N-1 contingency analysis associated with sudden changes in generation was also investigated in [17].

In this paper, a new method is proposed to determine the maximum generation output of a power plant taking into account the loading level of nearby high voltage transmission lines. When the power plant follows the proposed maximum generating set point, after any disturbance of some of the critical transmission lines, the power plant is able to provide generation that can reduce or even avoid the Expected Energy Not Supplied (EENS) cost. In this method, the offline N-1 contingency analysis is used to investigate the network security and indicate every possible contingency for the most sensitive and critical transmission lines. Then, all probable contingencies and operating scenarios are investigated carefully and the most cost effective output capacity of the plant with minimum EENS costs is determined.

The rest of the paper is organized as follows. Section II briefly highlights the N-1 contingency analysis method. Section III presents the proposed approach including three main steps of identifying the set of sensitive line, contingency analysis, the EENS calculation and the validation of the approach using a small test power system. Section IV provides a comprehensive investigation of the proposed approach when applied to a large Mashhad power generation plant in Iran.

\section{N-1 CONTINGENCY ANALYSIS}

One of the most important methods to investigate power system security is the contingency analysis. The safe operation of a power system is the key to a successful power system planning and management. To achieve the safe operation of power system, the operational constraints of the system must be identified. The contingency analysis is a useful tool to study different operational conditions of power systems. A contingency analysis investigates the outages of network 
elements or a sudden change in loads or generation to create a disturbed operating condition for the power system. These operating conditions are then used to identify the operational constraints of the system which are vital to be considered for the safe operation of the network. The location and the severity of the disturbance play an important role in the contingency analysis; so, contingency analysis usually considers disturbances in critical points of the system, which are highly probable or naturally unstable.

Moreover, studying different operating point of the network under the stressed condition will also leads us to the critical operating scenarios. Most of the disturbances during these critical stressed operating scenarios may lead the system toward instability or collapse. So, contingency analysis is a useful tool to assess the ability of a system to withstand cascading contingencies. Contingency analysis identifies the basis for preventive and corrective operational actions against extreme failures. Contingency analysis uses the current state of the system to identify possible series of component outages and vulnerable operational conditions.

The contingency analysis schemes are usually referred to as $\mathrm{N}-\mathrm{X}$ contingency analysis, where $\mathrm{N}$ is the total number of network components such as lines, generators and transformers, and $\mathrm{x}$ is the order of contingencies. $\mathrm{N}-\mathrm{x}$ contingency analysis requires the investigation of all possible permutations of $x$ outages. For example, an N-5 contingency analysis would evaluate all possible failures of up to five components collapsing together in a cascaded event. Due to the computational burden, the contingency analysis in this paper has been limited to N-1. However, the post event analysis of major outages has shown that the outage of a component usually leads to additional component outages in its neighborhood.

\section{THE PROPOSED METHOD}

The vast majority of power system failures are caused by transmission lines outages. Transmission lines outages in a power system may be due to a variety of reasons under different operating scenarios. However, in some cases, a disturbance in a transmission system may limit the output power of a power plant. In this circumstance, the generating plant may be forced to reduce its output power by disconnecting one of its generating units from the grid, which will be highly costly for the power plant; because, in addition to the significant cost of the generator startup, turbine fuel, cooling system and etc., the plant reduces its ability to provide a the load demand of the system to the costumers and needs to pay the EENS cost. To escape the cost of the EENS, the plant prefers to operate at optimal generating set points in which it faces zero EENS costs.

This paper presents a new method to determine the maximum generation output of a power plant to reduce or even avoid the EENS cost following the outages of some critical transmission lines. If the power plant generates the proposed maximum generation capacity, the effect of the disturbances on some of the critical transmission line on the output power of the plant can be minimized. This method investigates all available network transmission lines and every possible disturbance that can lead to a transmission line outage. Moreover, it can be implemented on all power plants located in a non-expandable transmission system area such as city centers. The novel proposed method follows the following stages:

1) Determine the set of the most sensitive lines using the sensitivity function in the neigborhood of the power plant.

2) Disconnect each line in the set of most sensitive lines and analyze its effect on the overloading of the nearby transmission system.

3) Calculate the EENS, considering the maximum generating point for the power plant.

For a better understanding of the proposed approach, each step of the method is implemented on a small hypothetical test system as shown in Fig. 1. To assure the balance between generation and demand, an infinite bus is also considered in test system referred to as the external system.

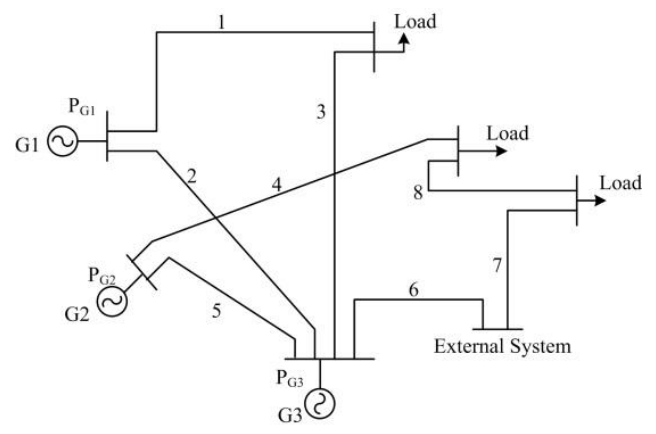

Figure 1. Single line diagram of the test system

In this section, the EENS will be calculated for each output capacity of G1 (the power plant of interest) using the proposed method. The following subsections present the steps of the proposed method.

\section{A. The Set of the Most Sensitive Lines}

To determine the set of the most sensitive lines in the test system, the sensitivity of the lines loading level to the output power of the power plant of interest (G1 in this example) is calculated using (1),

$$
S_{i}=\frac{\Delta I_{\text {Line }_{i}}}{\Delta P}
$$

where $S_{i}$ is the sensitivity of $i^{t h}$ line, $\Delta I_{\text {Line }}$ is the change in the transmission line current and, $\Delta P$ is the change in the generator active power.

To access the lines current and the plant output power, an AC load flow is performed, while initially the generation of the power plant of interest (G1) is set to zero. Then the active power output the plant (G1) is increased step by step to its rated value. The magnitude of each increasing step is $\Delta P$. Increasing the output power of G1 increases the loading level of some lines. In each step, the change in each of the line current is calculated using (2),

$$
\Delta I_{\text {Line }}^{j}=I_{\text {Line }}^{j}-I_{\text {Line }}^{j-1}
$$

where $\Delta I_{\text {Line }}$ is the change in the transmission line current, $I_{\text {Line }}$ is the line current at $j^{t h}$ step and $I^{j-1}$ Line is the line current at $j-1^{\text {th }}$ step. 
Considering $\triangle P$ and (2), at each step the sensitivity factor of each line is calculated using (1). Then, the lines with the highest values of sensitivity $\left(S_{i}\right)$ will form the set of most sensitive lines. In the considered test system, lines 1, 2 and 4 are the most sensitive lines with the highest value of sensitivity $\left(S_{i}\right)$, and they form the set of most sensitive lines.

\section{B. Contingency Anlysis Using the Set of Most Sensitive Lines}

The set of most sensitive lines includes the lines which loading levels are highly influenced by the power plant output power. These lines are the best candidates to perform the N-1 contingency analysis. For each step $\left(P_{G 1}+\Delta P\right)$, these lines will be disconnected one by one; and, for each unique disconnected line, the AC power flow is performed, and, the loading level for the rest of the transmission lines is identified. If the line outage does not overload transmission system, the mentioned line will be skipped and the next line in the set will be disconnected. But, if the line outage produces overload in one or some of the other lines, there are two possibilities:

1) The line outage causes overload at all steps of the power plant generation increase. In this situation, the power plant output power has no influence on the overloading of the other lines, when the mentioned line is disconnected; so, this line will be eliminated from the set of the most sensitive lines.

2) The line outage causes overload after the generation output of the power plant (G1) increases by $N \times \Delta P$; in this case, the output power of $(N-1) \times \Delta P$, will be considered as the maximum generating power capacity of the specified unit. Here $N=P_{\text {Rated }} / \Delta P$ is the number of steps and $P_{\text {Rated }}$ is the rated output power of the power plant of interest. If the plant only generates this maximum capacity, any disturbance on the lines from the set of most sensitive lines, will not be able to reduce its generating output.

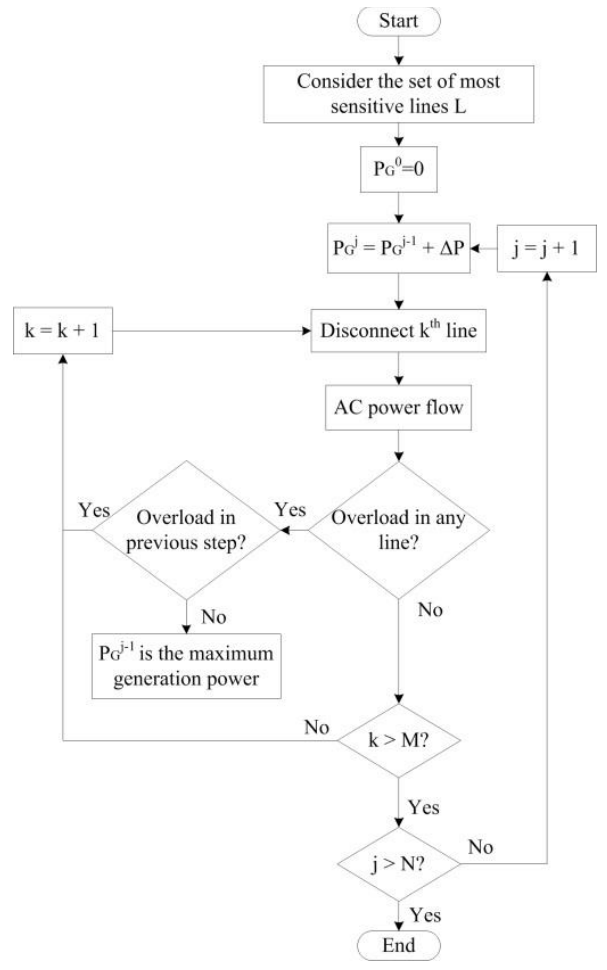

Figure 2. Flowchart of the proposed method
The flowchart of the proposed method is presented in Fig. 2. In this flowchart, each step of the increase in the power plant generation is indicated by $j=1,2, \ldots, N$ and $L=\left\{L_{1}, L_{2}, \ldots, L_{k}\right\}$, the set of most sensitive lines, where $k=1,2, \ldots, M$ indicate lines. Here, $M$ represents the total the number of lines.

The flowchart of the proposed method, shown in Fig. 2, is implemented on a small test power system presented in Fig. 1 and, the step by step results for the power plant of interest, G1, are presented in Table I. In Table I, the set of sensitive lines found earlier (lines 1, 2 and 4) in Section III-A, are disconnected one by one, as shown in the flow chart, and the overloading of the other transmission lines are monitored. In each step, the output active power of the power plant, G1, is increased by a fixed value of $\Delta P$.

TABLE I: LOADING OF THE TRANSMISSION SYSTEM AFTER EACH STEP OF INCREASE IN THE PlANT GENERATION OUTPUT

\begin{tabular}{|c|l|c|c|c|c|c|c|}
\hline \multicolumn{2}{|c|}{$\boldsymbol{P}_{\boldsymbol{G I}}$} & $\mathbf{0}$ & $\boldsymbol{A P}$ & $\mathbf{2 \Delta P}$ & $\mathbf{3 \Delta P}$ & $\mathbf{4 \Delta P}$ & $\mathbf{5 \Delta P}$ \\
\hline \multicolumn{2}{|c}{$\begin{array}{c}\text { Overload when no line is } \\
\text { disconnected }\end{array}$} & No & No & No & No & No & No \\
\hline $\begin{array}{c}\text { Overload when each } \\
\text { of the following lines } \\
\text { is disconnected }\end{array}$ & Line1 & No & No & No & Yes & Yes & Yes \\
\cline { 2 - 9 } & Line4 & Yes & Yes & Yes & Yes & Yes & Yes \\
\hline
\end{tabular}

Table I shows that in step 0 , if line 1 or line 4 is disconnected there will be no line overload in the transmission system, but if line 2 is disconnected, there will be an overload in one or some of the other lines. Further, Table I shows that if line 2 is disconnected, at each step of increase in the power plant output power, one or some of the other lines will be overloaded. In other words, any disturbance that leads to the outage of line 2 will cause overload in at least one other transmission line; consequently, this overloaded condition is not related to the generation output of the G1 and line 2 should be removed from the set of the most sensitive lines.

In the case of line 1 outage, the system behaves differently; in the first two steps, line 1 outage does not cause any overload in the transmission system but at step 3, it overloads the other transmission systems. So, the maximum output power of G1 should be limited to $P^{2}{ }_{G l}$, so that the system will not face any transmission line overload even if line 1 is disconnected. Similarly, for line 4, the outage of this line will overload the other transmission lines at step 5, hence, the maximum generation output of G1 based on line 4 outage, should be limited to $P_{G I}^{4}$. However, since the maximum output of G1 for an outage in line 1, is lower when compared with that due to line 4 outage; the maximum output power of G1 should be $P_{G I}{ }^{M a}$ equals to $P^{2}{ }_{G l}$, which is $2 \Delta P$. With this maximum output power of the power plant of interest, any outage in the set of most sensitive transmission lines, will not overload any of the transmission lines. However, it is to be noted that the value of $P_{G I} M a x$, is directly dependent on the system operating scenario; in other words, if the load demand of the system changes, the value of the $P_{G I}{ }^{M a x}$ will be changed accordingly.

\section{EENS Calculation}

For the small test system presented in Fig. 1, the maximum output active power of the power plant of interest, G1, is $P_{G I}{ }^{M a x}$, which is equal to $2 \triangle P$. The maximum output power of 
G1 will change for different values of the load demand from the system, and the load demand of the nearby network changes through a year constantly, for different seasons and even for different parts of the day. To consider the load variation in the network, the Load Duration Curve (LDC) is considered; it indicates the variation of the total load demand of a specific network in a downward form such that the greatest load is plotted in the left and the smallest one in the right. By using the LDC of a specific area, the required generation capacity of that area is determined. Here, a hypothetical LDC is allocated for the small test system in Fig. 1 and presented in Fig. 3.

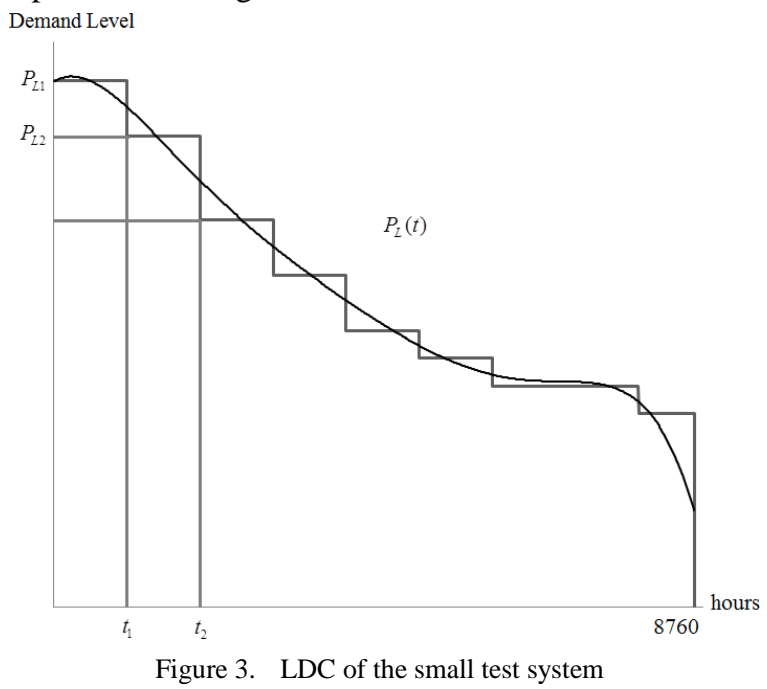

In Fig. 3, the horizontal axis varies from 0 to 8760 units which represents the hours throughout a year and the vertical axis shows the total loading capacity of the system. Using this curve, the total loading level of the system, in a specific period of time throughout a year, will be determined. In this regard, the system total loading (LDC) should be divided into the desired distinct time intervals and in each interval, the value of the system load in determined to be $P_{L}$. Let's consider 'n' as the number of time intervals through a year. The loading demand of the $l^{\text {th }}$ time interval is calculated as (3),

$$
P_{L l}=\int_{t_{l-1}}^{t_{l}} P_{L}(t) d t / t_{l} \quad l=1,2, \ldots, n
$$

where $P_{L}(t)$ is the LDC equation, $t_{l}$ is the $l^{\text {th }}$ time interval and $P_{L l}$ is the loading demand in the $l^{\text {th }}$ time interval.

As the number of intervals increases, the accuracy of the results will increase. After determining the load demand of each time interval, the Expected Energy Not Supplied (EENS) for the $l^{\text {th }}$ loading demand for the power plant of interest, $G n$, can be calculated using (4),

$$
\operatorname{EENS}_{l}=\left[\sum_{j=1}^{N}\left(P_{G n}^{j}-P_{G n}^{M a x}\right) p_{j}\right] t_{l}
$$

where $E E N S_{l}$ is the EENS for the $l^{\text {th }}$ loading demand interval, $P_{G n}{ }^{j}$ is the generation power of the $n^{\text {th }}$ power plant at $j^{\text {th }}$ step, $P_{G n}{ }^{M a x}$ is the maximum generation point of the $n^{\text {th }}$ power plant, which is calculated using the technique described in Section III-B, and $p_{j}$ is the summation of lines outage probability regarding to the generation of the specified unit. Similar method can be used to calculate the EENS for the other power plants of interest.

In (4), the only unknown parameter is $p_{j}$. Here, $p_{j}$ is calculated considering the generation of the unit in addition to the number of lines which will restrict its power injection into the grid. In other words, $p_{j}$, shows the summation of line outage probability for which their outage causes restriction in power transmission from the unit with $P_{G}{ }^{j}$ amount of generating power. Equation (5) shows the way by which $p_{j}$ is calculated,

$$
p_{j}=\sum_{m=1}^{l_{j}} p_{L_{m}}
$$

where $l_{j}$ is number of transmission lines for which their outage overloads the transmission system, and, $p_{L m}$ is the outage probability of $m^{\text {th }}$ line and it is provided by the utility.

It is preferred to report the annual value of the EENS for a power plant, since the LDC represents the total load demand of the system for a year. The EENS for a year is calculated using (6) and presented as $E E N S_{\text {Total }}$ as follows,

$$
E E N S_{\text {Total }}=\sum_{l=1}^{n} E E N S_{l}
$$

Assume that the LDC in Fig. 3 represents the total loading duration curve for the power system in Fig. 1. Considering the LDC equation in (3), the loading demands of the test power system in the first and second time interval are $P_{L 1}$ and $P_{L 2}$, as shown in Fig. 3 respectively. The values of $p_{j}$ for different generation capacity of G1 in the test system given in Fig. 1, are calculated in each step using (5) and presented in Table II considering the value of $P_{G I}$ Max equals $2 \Delta P$, where the total

\begin{tabular}{|c|c|c|c|}
\hline Step & $P_{G 1}^{j}$ & $p_{j}$ & $\left(P_{G 1}^{j}-P_{G 1}^{M a x}\right) p_{j}$ \\
\hline Step 1 & $P_{G 1}^{1}=\Delta P$ & $p_{1}=0$ & $(-\Delta P) 0$ \\
\hline Step 2 & $P_{G 1}^{2}=2 \Delta P$ & $p_{2}=0$ & $(0) 0$ \\
\hline Step 3 & $P_{G 1}^{3}=3 \Delta P$ & $p_{3}=p_{\text {Line } 1}$ & $(\Delta P) p_{\text {Linel }}$ \\
\hline Step 4 & $P_{G 1}^{4}=4 \Delta P$ & $p_{4}=p_{\text {Line } 1}$ & $(2 \Delta P) p_{\text {Line } 1}$ \\
\hline Step 5 & $P_{G 1}^{5}=5 \Delta P$ & $p_{5}=p_{\text {Line } 1}+p_{\text {Line } 4}$ & $(3 \Delta P)\left(p_{\text {Line } 1}+p_{\text {Line } 4}\right)$ \\
\hline Step 6 & $P_{G 1}^{6}=6 \Delta P$ & $p_{6}=p_{\text {Line } 1}+p_{\text {Line } 4}$ & $(4 \Delta P)\left(p_{\text {Line } 1}+p_{\text {Line } 4}\right)$ \\
\hline
\end{tabular}
load demand. $\mathrm{P}_{L 1}$, is as used in Table $\mathrm{I}$.

TABLE II: The EENS Results of THE PROPOSED METHOD ON THE TEST POWER SYSTEM

It is obvious that when the output of the power plant is set to $P_{G I}^{M a}$ or $2 \Delta \mathrm{P}$ for the load demand $\mathrm{P}_{\mathrm{L} 1}$ used previously in Table I, the total EENS will be 0, however higher values of the power plant output will lead to EENS and therefore the plant will have to pay for the EENS cost.

\section{CASE STUdy}

The proposed method is implemented on Mashhad power plant in Iran to reduce the cost of EENS as much as possible. Mashhad power plant was installed more than 40 years ago and currently it has seven operating generation units. Except for the smallest generation unit, six units are connected to the Khorasan Regional Electric Network (KREN) by a $63 \mathrm{kV}$ 
substation. The nominal capacity of the Mashhad power plant is 367MVA. The transmission system, around Mashhad power plant, is modeled precisely as presented in Fig. 4.

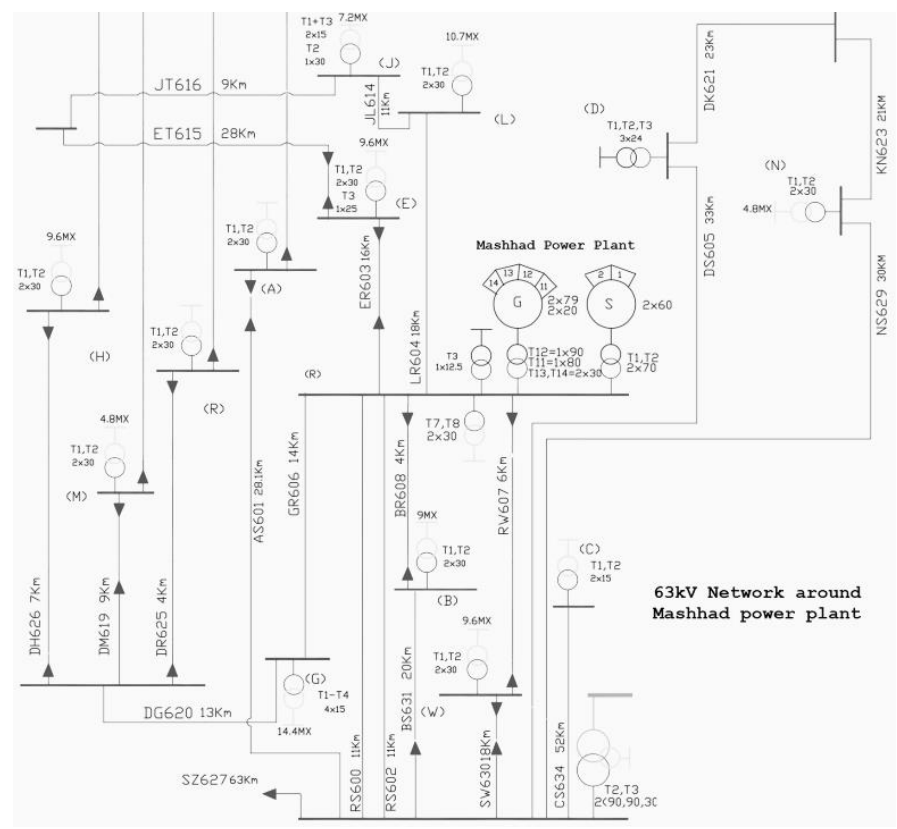

Figure 4. The $63 \mathrm{kV}$ network around Mashhad power plant

Due to the city expansion after 40 years, the power plant is currently located in the middle of the commercial zone in the city center with dramatically increasing load demand, without the possibility of any transmission system expansion. As a result, most of the times, the power plant generates less than its rated value. The proposed method is used to determine the maximum output power of the plant, when any single disturbance on some of the critical transmission lines do not produce EENS cost for the plant. To model KREN, only $400 \mathrm{kV}, 230 \mathrm{kV}, 132 \mathrm{kV}$ and $63 \mathrm{kV}$ substations are considered, and the rest of the network is modeled as constant current loads connected to these substations. The LDC curve for the KREN is also obtained for the year of 2012 and presented in Fig. 5. Here, the LDC curve is divided into three district time intervals which represented by $H$ for High load demand, $M$ for Medium load demand and $L$ for Low load demand.

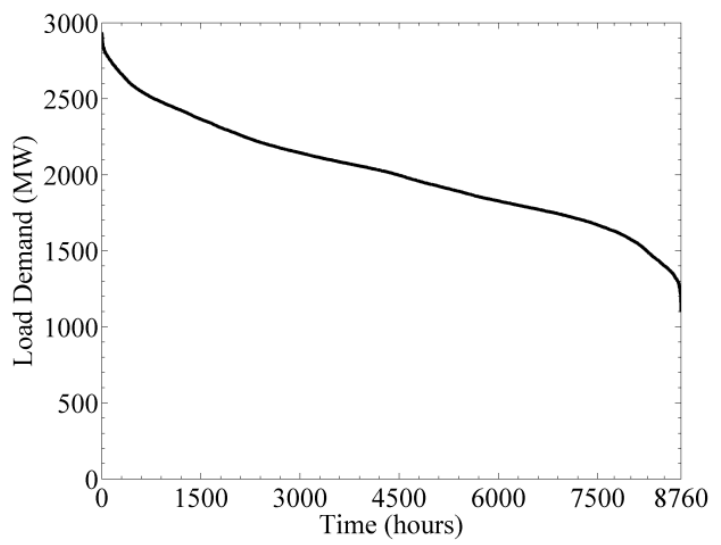

Figure 5. KREN LDC for 2012

The high load demand area covers the loads between the maximum value and $70 \%$ of the maximum value, the medium load demand area covers the loads which are between $40 \%$ and $70 \%$ of the maximum load and the low load demand area covers the loads which are less than $40 \%$ of the maximum load. Table III shows the detailed characteristics of the three load intervals.

TABLE III: THE CHARACTERISTICS OF EACH LOAD INTERVAL

\begin{tabular}{|c|c|c|c|}
\hline Load demand interval & $\boldsymbol{H}$ & $\boldsymbol{M}$ & $\boldsymbol{L}$ \\
\hline Total load (MW) & 3128 & 2623 & 2183 \\
\hline Total time (Hours) & 1590 & 5260 & 1911 \\
\hline Loading time percent (\%) & 18.1 & 60 & 21.9 \\
\hline
\end{tabular}

The simulation is performed using the DIgSILENT PowerFactory software. The following assumptions were considered in this study to reduce the computational complexity.

1) The probability of simultaneous outage of transmission lines is zero.

2) The outage probability of all lines is equal.

3) The outage probability of bus-bars is zero.

The proposed method is applied to each load demand intervals. For example, the proposed method will be applied to high load demand interval of Mashhad power plant and the results are summarized in Table IV. First the most sensitive lines are identified. Eleven lines from the transmission system around Mashhad power plant, which are presented in Table IV, are designated to form the set of the most sensitive lines. After considering the set of the most sensitive lines, the output power of Mashhad power plant is increased step by step and the overloading condition of one or some of the transmission lines in the system is obtained.

TABLE IV: THE LOADINGCONDITION OF THE TRANSMISSION SYSTEM AROUND MASHHAd POWER Plant IN SIX OPERATING SCENARIOES

\begin{tabular}{|c|c|c|c|c|c|c|c|}
\hline \multicolumn{2}{|c|}{ Plant generation (MW) } & $\mathbf{2 0 0}$ & $\mathbf{2 4 0}$ & $\mathbf{2 8 0}$ & $\mathbf{3 0 0}$ & $\mathbf{3 2 0}$ & $\mathbf{3 4 0}$ \\
\hline $\begin{array}{c}\text { Overload when no line is } \\
\text { disconnected }\end{array}$ & No & No & No & No & No & Yes \\
\hline \multirow{4}{*}{$\begin{array}{c}\text { Overload when each } \\
\text { of the following lines } \\
\text { is disconnected }\end{array}$} & ER603 & Yes & Yes & Yes & Yes & Yes & Yes \\
\cline { 2 - 9 } & ET615 & Yes & Yes & Yes & Yes & Yes & Yes \\
\cline { 2 - 8 } & GR606 & No & No & No & Yes & Yes & Yes \\
\cline { 2 - 8 } & RS602 & No & No & No & No & No & Yes \\
\cline { 2 - 8 } & BR608 & No & No & No & No & Yes & Yes \\
\cline { 2 - 8 } & LR604 & Yes & Yes & Yes & Yes & Yes & Yes \\
\cline { 2 - 8 } & BS631 & No & No & No & No & No & Yes \\
\cline { 2 - 8 } & JT616 & Yes & Yes & Yes & Yes & Yes & Yes \\
\cline { 2 - 8 } & DG620 & No & No & No & No & No & Yes \\
\cline { 2 - 8 } & JL614 & No & No & No & No & No & Yes \\
\hline
\end{tabular}

The result from Table IV shows that one or some of the transmission system is overloaded when the output active power of the Mashhad power plant is 340MW. Consequently, due to the restricted transmission system around the power plant, it will not be able to supply its nominal output power (376MW) in the $H$ loading interval. Moreover, when lines ER603, ET615, LR604 and JT616 are disconnected, increasing the output power of the power plant does not influence the overload condition of the transmission system. So, these lines should be eliminated from the set of the most sensitive lines, since, any disturbance in these lines will overload the transmission system, irrespective of the output power of the Mashhad power plant. 
However, the outage in the GR606 and RS600 overloads the transmissions system when the output power of the plant reaches $300 \mathrm{MW}$ and $320 \mathrm{MW}$ respectively. In this case of GR606, the transmission system is overloaded sooner than the RS600; so, using this result from the n-1 contingency analysis, the maximum output power of the power plant is determined as 280MW. With this maximum output power, the Mashhad power plant will not face any avoidable EENS cost if a disturbance happens in the nearby transmission system. The same procedure is performed for the medium and low loading level of the system around Mashhad power plant and the maximum generation point of Mashhad power plant for high, medium and low load demand interval is $280 \mathrm{MW}, 310 \mathrm{MW}$ and $320 \mathrm{MW}$ respectively. Using $4 \%$ as the probability of the line outage in the $63 \mathrm{kV}$ transmission system around Mashhad power plant, the values of EENS for different output powers of Mashhad power plant are calculated and presented in Fig. 6.

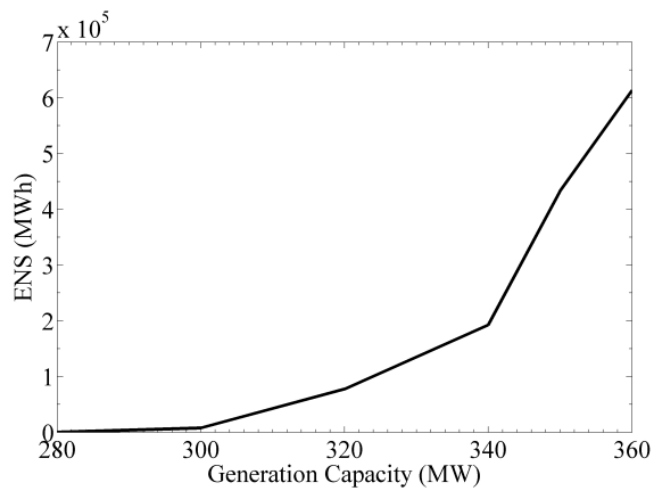

Figure 6. The EENS of Mashhad power plant for a year in the high load demand

Fig. 6 shows the values of the EENS as the generation output of Mashhad power plant increases. It is obvious that when the output power of Mashhad power plant is $280 \mathrm{MW}$, the value of EENS is zero, but as the output power increases to $300 \mathrm{MW}$, the EENS is $7709 \mathrm{MWh}$; and, when the output power increases by $20 \mathrm{MW}$ and reaches $320 \mathrm{MW}$, the value of EENS reaches 77680MWh, almost 10 times of the EENS when the output power was $300 \mathrm{MW}$.

\section{CONCLUSION}

The inconsistency between the transmission system expansion and the increase in load demand will have the effect of restricting the output of power plants significantly; specially, when the generation units are located at the urban areas. If the output of the power plant is not set correctly, some of the output cannot flow through the transmission lines, resulting in 'trapped' energy in the power plant. This can lead to the plant having to pay the EENS cost. To avoid the cost of the EENS, the power plant should limit its generation to a maximum capacity, in which a disturbance in the transmission lines will not 'trap' the generation inside the power plant. In this paper, a new method is proposed to determine this maximum capacity of the power plant. This new method is based on the N-1 contingency analysis; where only a maximum of one transmission line outage in the transmission system is considered in this study. The proposed method is applied to Mashhad power plant in the middle of Mashhad city in Iran. The results indicate the amount of EENS cost for the most probable output power of the plant. Using the result, the power plant is able to choose its maximum generation capacity that can produce no EENS cost and therefore increasing the power plant revenue.

\section{REFERENCES}

[1] Billinton, R., and Allen, R., Reliability Evaluation of Power Systems, 2nd ed., New York: Plenum Press, 1996.

[2] Chowdhury, A. A., and Koval, D. O., "Quantitative transmission system reliability assessment", IEEE Transactions on Industry Application, vol. 46, no. 1, January 2010.

[3] Billinton R., and Li, W., Reliability assessment of electric power systems using Monte Carlo methods, New York: Plenum Press, 1994.

[4] H. Zhang, V. Vittal, G. T. Heydt, and J. Quintero, "A mixed-integer linear programming approach for multi-stage security-constrained transmission expansion planning," IEEE Trans. Power Syst., vol. 27, no. 2, pp. 1125-1133, May 2012.

[5] Kim J. H., Park J. B., Park J. K. and Joo S. K., "A market-based analysis on the generation expansion planning strategies," Intelligent Systems Application to Power Systems, 2005. Proceedings of the 13th International Conference on, 6-10 Nov. 2005.

[6] Kim S. K., Park J. H., Lee H. C., Park G. P., Lee S. S., Kim W. and Yoon Y. T., "Comparison between centralized planning model and market oriented planning model for the generation expansion plan", Power and Energy Society General Meeting, IEEE, 2010.

[7] El-Sayed M. A. H., "Reliability modeling for expansion-planning of electric-power plants", Reliability, IEEE Transactions on, vol. 40, no. 3, August 1991.

[8] Gurpa R. and Goel L., "System planning utilizing value based reliability approach", PowerCon 2004, vol. 2, pp. 1981 - 1985, November 2004.

[9] Porkar S., F. Firuzabad M., A. T. Fard A. and Porkar B., "An approach to determine spinning reserve requirements in deregulated electricity market", PSCE '06. 2006 IEEE PES, pp. 1341-1344, 2006.

[10] Zhong J. and Wu F. F., "Operating reserve value at risk", Power Engineering Society General Meeting, 2006.

[11] Condren, J.; Gedra, T.W.; Damrongkulkamjorn, P., "Optimal power flow with expected security costs," Power Systems, IEEE Transactions on, vol. 21, no. 2, pp. 541-547, May 2006.

[12] Mishra, V.J.; Khardenvis, M.D., "Contingency analysis of power system," Electrical, Electronics and Computer Science (SCEECS), 2012 IEEE Students' Conference on, vol. 1, no. 4, pp. 1-2, March 2012.

[13] Arroyo, J.M., "Bilevel programming applied to power system vulnerability analysis under multiple contingencies," Generation, Transmission \& Distribution, IET, vol.4, no.2, pp.178-190, Feb. 2010.

[14] C. M. Davis and T. J. Overbye, "Multiple element contingency screening," IEEE Trans. Power Syst., vol. 26, no. 3, pp. 1294-1301, Aug. 2011.

[15] A. Street, F. Oliveira, and J. M. Arroyo, "Contingency-constrained unit commitment with - security criterion: A robust optimization approach," IEEE Trans. Power Syst., vol. 26, no. 3, pp. 1581-1590, Aug. 2011.

[16] Chen, R.L.-Y.; Cohn, A.; Neng Fan; Pinar, A., "Contingency-Risk Informed Power System Design," Power Systems, IEEE Transactions on, vol. 29, no. 5, pp.2087-2096, 2014.

[17] Papaefthymiou, G.; Verboomen, J.; Schavemaker, P.H.; van der Sluis, L., "Impact of Stochastic Generation in Power Systems Contingency Analysis," PMAPS 2006. International Conference on, vol. 1, no. 6, pp. 11-15, June 2006.

[18] Amjady N., Esmaili M., "Application of a new sensitivity analysis framework for voltage contingency ranking", Power Systems, IEEE Transactions on, vol. 20, , no. 2, pp. 973-983, May 2005.

[19] Flueck, A.J., Gonella, R., Dondeti, J.R., "A new power sensitivity method of ranking branch outage contingencies for voltage collapse", Power Systems, IEEE Transactions on, vol. 17, no. 2, pp. 265-270, May 2002.

[20] Ruiz P. A., Sauer P. W., "Voltage and reactive power estimation for contingency analysis using sensitivities", Power Systems, IEEE Transactions on, vol. 22, no. 2, pp. 639-647, May 2007.

[21] Guler T., Gross G., and Liu M., "Generalized line outage distribution factors", Power Systems, IEEE Transactions on, vol. 22, no. 2, pp. 879881, May 2007. 